\title{
Technè
}

La science au service de l'histoire de l'art et de la préservation des biens culturels

48 | 2019

Les couleurs de l'Antique

\section{La peinture sur les sols de mortier et les mosaïques d'époque hellénistique : modes d'emploi d'une polychromie architecturale largement méconnue}

\section{Anne-Marie Guimier-Sorbets}

\section{(2) OpenEdition}

1 Journals

Édition électronique

URL : https://journals.openedition.org/techne/1956

DOI : 10.4000/techne.1956

ISSN : 2534-5168

Éditeur

C2RMF

\section{Édition imprimée}

Date de publication : 31 décembre 2019

Pagination : 27-33

ISBN : 978-2-11-152831-4

ISSN : $1254-7867$

\section{Référence électronique}

Anne-Marie Guimier-Sorbets, «La peinture sur les sols de mortier et les mosaïques d'époque hellénistique : modes d'emploi d'une polychromie architecturale largement méconnue », Technè [En ligne], 48 | 2019, mis en ligne le 01 juillet 2021, consulté le 03 avril 2022. URL : http:// journals.openedition.org/techne/1956 ; DOI : https://doi.org/10.4000/techne.1956

\section{cc) (ㅇ) $\ominus$}

La revue Technè. La science au service de l'histoire de l'art et de la préservation des biens culturels est mise à disposition selon les termes de la Licence Creative Commons Attribution - Pas d'Utilisation Commerciale - Pas de Modification 4.0 International. 
Anne-Marie Guimier-Sorbets

\section{La peinture sur les sols de mortier et les mosaïques d'époque hellénistique: modes d'emploi d'une polychromie architecturale largement méconnue}

Painting on mortar floors and mosaics in the Hellenistic period: techniques of a little-known architectural polychromy

Résumé. En nous fondant sur des pavements découverts en Macédoine, à Rhodes, à Délos, à Alexandrie et dans quelques autres sites du monde grec, nous nous attacherons à montrer comment la polychromie a été mise en æuvre et développée sur les sols de mortier et les mosä̈ques à partir des $V^{e}-I V^{e}$ siècles avant J.-C., dans des contextes tant publics que domestiques. Le premier objectif était de décorer les sols par l'adjonction de couleurs vives pour étendre et aviver la polychromie, élément important du décor architectural grec. Le second était de copier la peinture murale ou de chevalet, art majeur de cette époque, lui-même fortement polychrome et illusionniste. En raison de l'altération de la peinture et des matériaux artificiels employés, cette polychromie n'a pas été repérée au moment de la découverte et reste en grande partie à restituer.

Mots-clés. Art grec, maisons, palais, époque hellénistique, polychromie des sols, mosaïque, verre, faïence, opus tessellatum, opus vermiculatum.
Abstract. Basing our research on pavements found in Macedonia, Rhodes, Delos, Alexandria and a few other sites in the Greek world, we shall strive to show how polychromy was employed and developed on mortar floors and mosaics from the 5th and 4th centuries B.C. onwards, in both public spaces and private homes. The first objective was to decorate the floors by adding bright colours to extend and enliven the polychromy, an important feature of Greek architectural decor. The second was to copy mural or easel painting, a major art form in this period, itself highly colourful and illusionistic. Owing to the deterioration of the paint and other artificial materials used, this polychromy failed to be spotted at the time of excavation and largely remains to be taken into account.

Keywords. Greek art, houses, palaces, Hellenistic period, polychrome floors, mosaic, glass, faience, opus tessellatum, opus vermiculatum.
Dans les maisons grecques d'époque classique et hellénistique, sols de mortier et mosaïques avaient pour fonction de rendre les sols imperméables - pour en faciliter l'entretien -, et de les décorer, surtout dans la salle de banquet (andron), pièce de réception et d'apparat du maître de maison. Les sols de mortier étaient blanchâtres car constitués essentiellement de chaux, tandis que les premières mosaïques étaient bichromes, le décor formé par des galets clairs se détachant sur un fond constitué de galets sombres, à l'imitation de la céramique contemporaine. L'adjonction de couleur (rouge et jaune) a permis d'anoblir ces sols et, certainement aussi, de mieux refléter la lumière dans ces espaces intérieurs aux ouvertures assez étroites ${ }^{1}$.

\section{Ajouter de la couleur à l'intérieur des bâtiments}

\section{Sols de mortier finement poncés et peints}

Lors des fouilles américaines d'Olynthe, dans le nord de la Grèce, les chercheurs avaient noté la présence de couleur rouge et jaune sur les sols de certains andrones, dans un quartier édifié et habité de 432 à 348 avant J.-C. ${ }^{2}$ Les sols de mortier de chaux, dont la surface était soigneusement poncée, pouvaient soit être recouverts uniformément d'une fine couche d'enduit de couleur rouge (comme à Alexandrie, dans une maison du terrain du Cricket Ground ${ }^{3}$ ), soit porter un décor peint, comme on l'a constaté notamment dans des édifices de Gaule : à Marseille, on a ainsi une bande de postes sur le sol d'une maison $\mathrm{du} \mathrm{III}^{\mathrm{e}}$ siècle avant J.-C. et une bande monochrome rouge sur celui d'une rotonde, dans un bain de type grec de la première moitié du même $\mathrm{III}^{\mathrm{e}}$ siècle avant $\mathrm{J}$.-C. ${ }^{4}$ Un troisième sol peint à décor géométrique a été découvert récemment lors des fouilles de la Verrerie de Trinquetaille à Arles, dans la maison de la Harpiste, datant de la première moitié du $\mathrm{I}^{\mathrm{er}}$ siècle avant J.-C. ${ }^{5}$ : il est en cours de nettoyage et d'étude dans l'atelier de restauration des mosaïques du musée départemental de l'Arles antique. Ce type de décor devait être relativement fréquent, mais il se conserve mal sur la surface du mortier dégradé par l'usage puis par les conditions d'enfouissement, et il doit être noté dès la mise au jour du sol. Dans les

Anne-Marie Guimier-Sorbets, professeur émérite à l'université Paris Nanterre UMR ArScAn (anne-marie.guimier-sorbets @ parisnanterre.fr). 


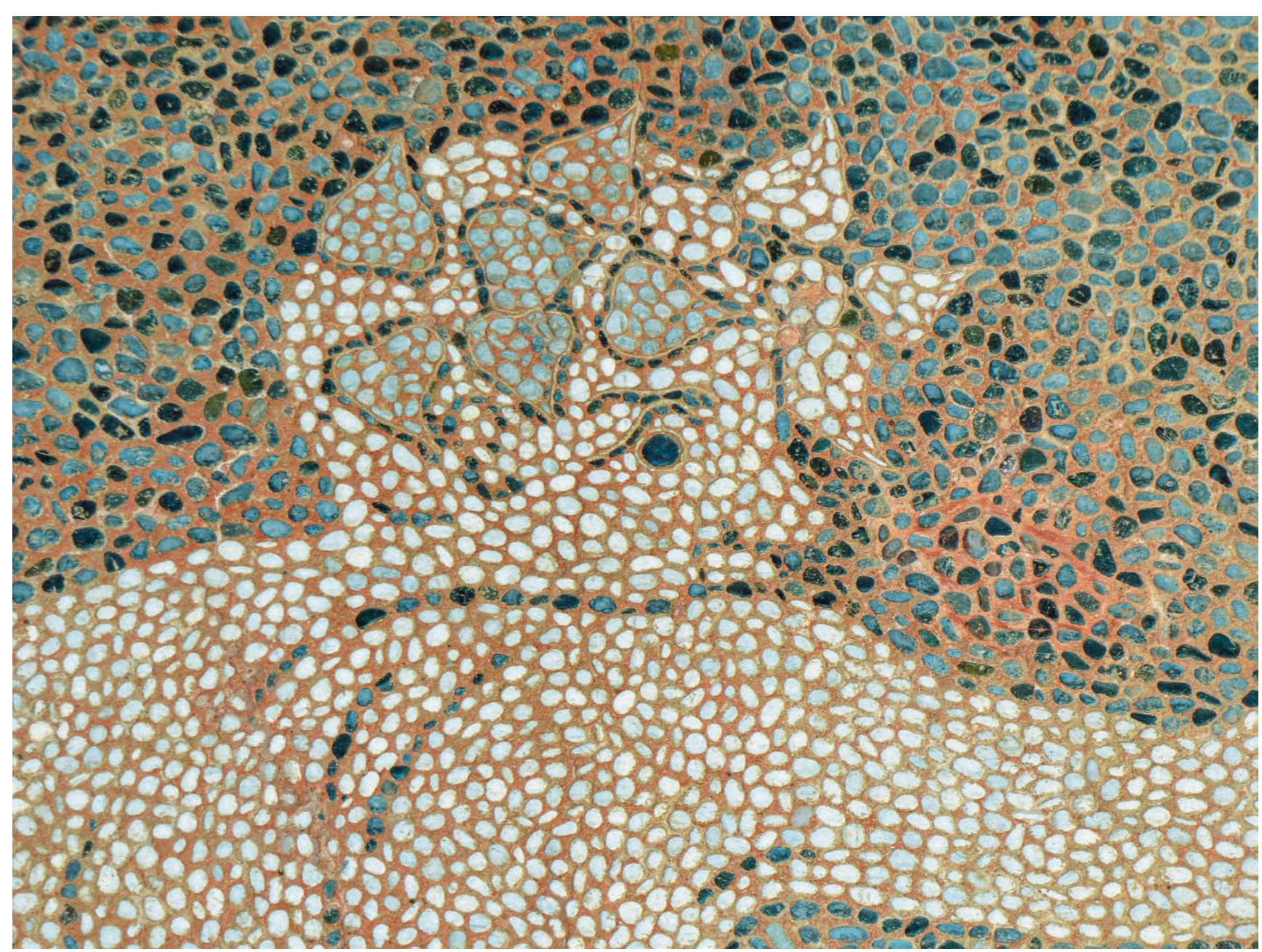

Fig. 1. Rhodes, musée archéologique, mosaïque au Centaure, détail. @ A.-M. Guimier-Sorbets/ArScAn.

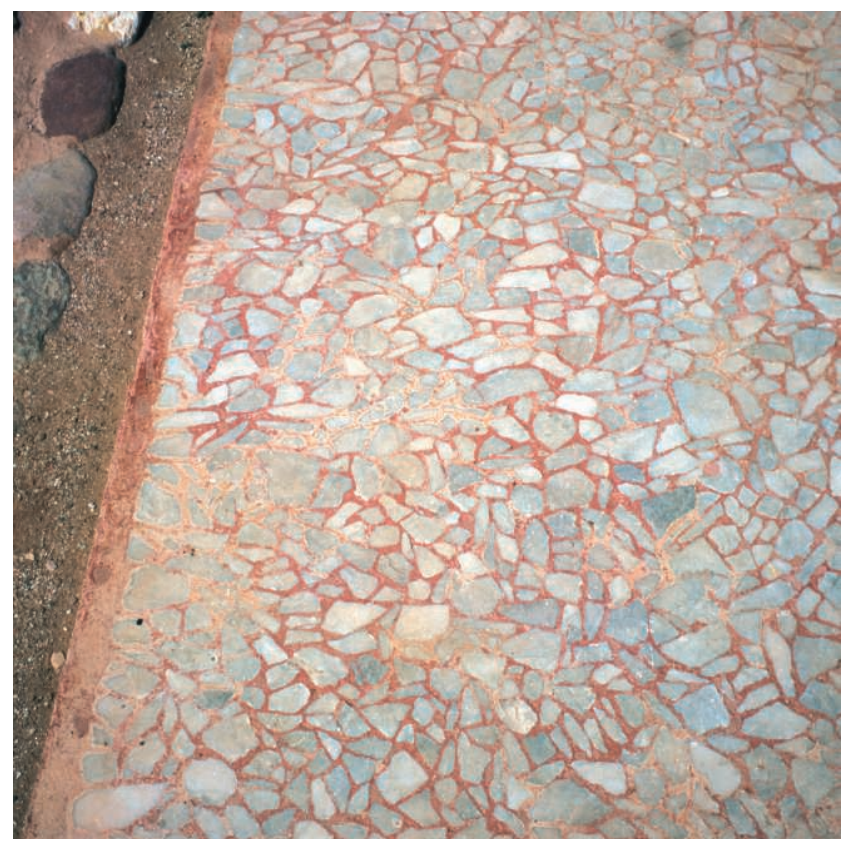

Fig. 2. Olynthe, mosaïque d'éclats de la maison 5. (C A.-M. GuimierSorbets/ArScAn. tombes du Nord de la Grèce (Korinos-Pydna) et de la Bulgarie actuelle (Kazanlak) ${ }^{6}$, les sols de mortier étaient fréquemment peints en rouge ; ils étaient plus rarement décorés d'un motif de damier polychrome, comme dans plusieurs tombes d'Amphipolis, toutes d'époque hellénistique ${ }^{7}$. Parmi les exemples donnés dans ce texte, seul un fragment de sol de la tombe de Korinos-Pydna, conservé au musée du Louvre, a pu être analysé. L'équipe du C2RMF a mis en évidence la présence d'une couche d'enduit blanchâtre (épaisse de $1 \mathrm{~cm}$ ), surmontée d'une couche $(1 \mathrm{~mm})$ colorée en rouge par l'hématite ; au-dessus, un badigeon de vermillon semble avoir été posé $a$ $\operatorname{secco}^{8}$. On voit ainsi le soin mis pour la coloration riche de ce sol de tombe très lisse et dont la surface est bien conservée.

\section{Mosaïques de galets et d'éclats de pierre fixés dans un mortier coloré dans la masse ou peint}

Lorsque le décor des premières mosaïques a été rendu par des galets de rivière clairs se détachant sur un fond sombre, à partir du v ve siècle avant J.-C., des mosaïstes ont gardé l'usage de peindre la surface du mortier interstitiel, comme on a pu 


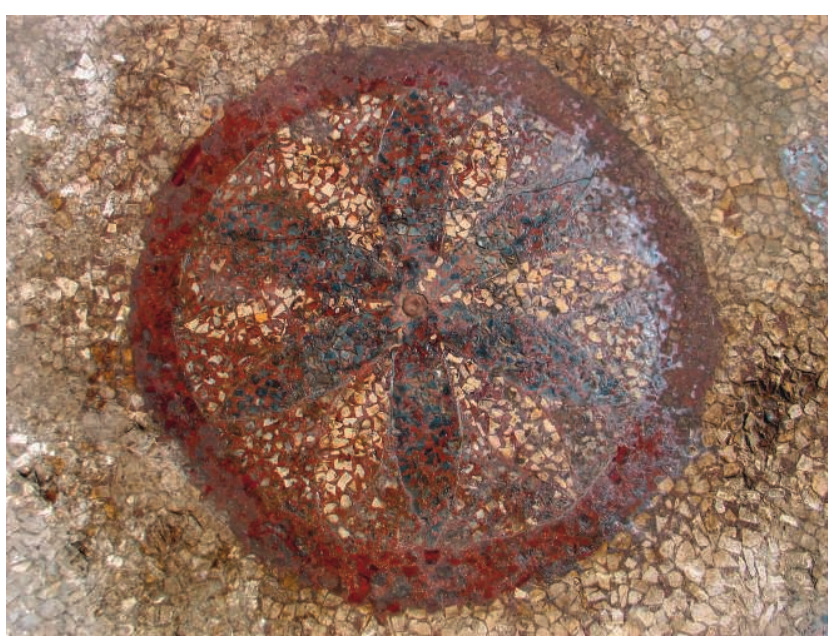

Fig. 3. Karnak, fleuron central de la tholos nord. (C) A.-M. GuimierSorbets/ArScAn.

le constater récemment sur des mosaïques de salles de banquet de Rhodes (première moitié du $\mathrm{III}^{\mathrm{e}}$ siècle av. J.-C.) exposées au musée archéologique de la ville. Sur le pavement au Centaure, l'ensemble du mortier est peint en rouge (fig. 1), tandis qu'une opposition de couleurs a été mise en ouvre pour le pavement au Bellérophon, contemporain du précédent : la surface du mortier y est colorée de rouge dans le tapis principal et de jaune dans le reste du pavement ${ }^{9}$.

La couleur rougeâtre des sols en béton de tuileau, parfois décorés de tesselles de pierre (opus signinum) était souvent ravivée d'une couche superficielle de peinture rouge vif. Fréquents dans la partie occidentale du pourtour méditerranéen (Italie, Gaule, Espagne), ces sols de béton de tuileau peint sont aussi attestés dans quelques maisons de Délos, comme dans celle de Fourni (Grèce) ${ }^{10}$.

Dans la maison 5 d'Olynthe, le sol de la salle de banquet était revêtu d'une mosaïque d'éclats de pierre blanche fixés dans un mortier dont la surface avait été peinte en rouge, comme on peut le constater après la restauration du sol laissé in situ ${ }^{11}$ (fig. 2). L'emploi de cette technique produit un sol assez luxueux, qui est également attesté dans des salles de banquet ou d'apparat du palais des rois de Macédoine à Vergina $^{12}$ (Grèce), comme sur le sol de la tombe aux Caryatides d'Amphipolis ${ }^{13}$. Cette même technique se retrouve à Délos, et même dans un bain grec découvert en HauteÉgypte devant le pylône du sanctuaire d'Amon à Karnak ${ }^{14}$ (fin III $^{\mathrm{e}}$-première moitié du $\mathrm{II}^{\mathrm{e}}$ siècle av. J.-C.). Les sols de certaines pièces y sont décorés par la juxtaposition de cette technique blanche et rouge avec des parties en mortier coloré uniformément de rouge. Au centre de la rotonde nord, la plus finement décorée, une composition formée d'un fleuron central autour duquel rayonnent des animaux aquatiques (dauphins et tilapias du Nil) est réalisée en éclats de pierre de diverses couleurs fixés dans un mortier rouge (fig. 3). Ce décor peint était suffisamment solide pour résister à l'eau qui devait couvrir le sol durant l'utilisation de ces bains par affusion, lorsque des esclaves arrosaient d'eau chaude les baigneurs installés dans de petites baignoires en sabot. En ravivant la polychromie du sol, l'eau qui y était répandue devait à la fois évoquer le contexte aquatique et, en été, rafrầchir par évaporation l'atmosphère surchauffée de cet édifice balnéaire de Haute-Égypte. La conservation de tels sols polychromes exposés au soleil et aux vents de sable reste assez problématique.

Dans tous ces exemples, la coloration de la surface du mortier interstitiel n'a pas pour objectif de le cacher mais, bien au contraire, de le souligner en créant un effet de contraste avec les éléments de pierre qui y sont fixés. Dans une mosaïque faite de galets ou d'éclats de pierre, le mortier reste visible et sa coloration permet d'ajouter une vive polychromie complémentaire de celle, limitée, des matériaux naturels.

\section{Imiter la peinture et sa palette}

À partir du IV siècle avant J.-C., les peintres ont enrichi leur palette de façon importante : à la tétrachromie (noir, blanc, rouge et jaune, colores austeri) vantée par Pline (Histoire naturelle, XXXV, 50), ils ont ajouté de nombreuses couleurs vives

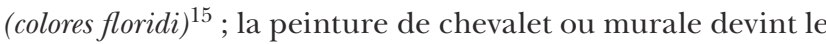
« grand art» que les mosaïstes ont voulu imiter, aussi bien dans le décor figuré, géométrique que dans le décor végétal. Ainsi l'adoption du style illusionniste (rendre l'illusion du volume dans un espace à deux dimensions) a nécessité la réalisation de dégradés d'une large gamme de couleurs. Cette volonté de transposer la peinture en mosaïque a eu une série de conséquences majeures pour l'évolution des pavements :

- le remplacement des galets et des éclats par des tesselles, éléments de pierre taillés de forme cubique (opus tessellatum) ou même de formes variées et de très petite taille (1-2 mm de côté) dans l'opus vermiculatum, dont les côtés devenaient ainsi quasi-jointifs,

- extension de la gamme des matériaux et adoption des matériaux artificiels comme le verre et la faïence, ainsi que des lames de terre cuite et de plomb,

- ajout de peinture en surface pour aviver la teinte des tesselles et colorer les joints de mortier de manière à effacer la discontinuité des matériaux, pourtant caractéristique de cet $\operatorname{artisanat}^{16}$.

Les mosaïques de galets de Pella, seconde capitale des rois de Macédoine, montrent déjà des innovations liées à la volonté de copier les peintures : l'adjonction de lames de terre cuite et de lames de plomb pour dessiner des lignes nettes au milieu des galets, mais aussi l'emploi de boules de faïence vert clair pour rendre le feuillage de la couronne et du thyrse du Dionysos, sur la mosaïque de la maison $\mathrm{I}, 1^{17}$. À partir de la fondation d'Alexandrie en 331 avant J.-C., les commandes des mêmes élites macédoniennes encouragent les mosaïstes à poursuivre leurs innovations. Dès la fin $\mathrm{du} \mathrm{IV}^{\mathrm{e}}$ siècle avant J.-C., on constate l'emploi d'opus tessellatum, puis celui de l'opus vermiculatum dans la mosaïque de Thmouis signée de 


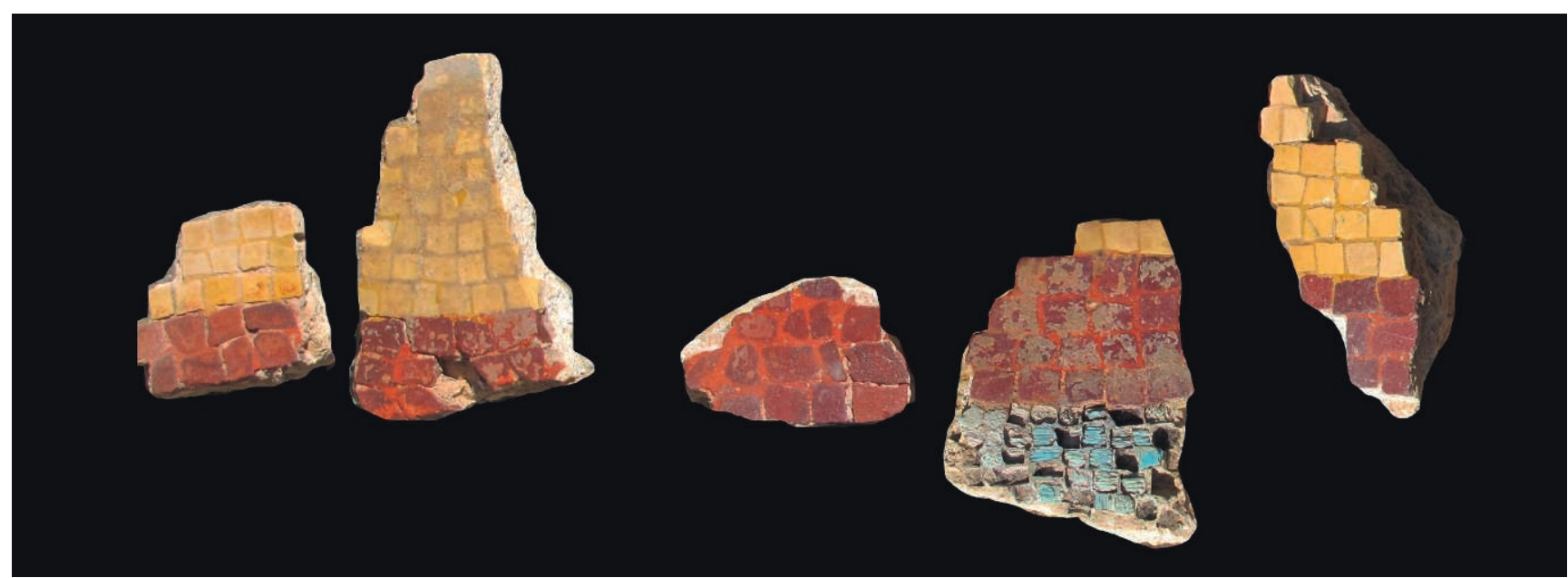

Fig. 4. Délos, fragments de mosaïque d'étage de la maison de Fourni. (C) A. Guimier/ArScAn.

Sophilos (vers 200 av. J.-C.), et dans l'autre panneau circulaire, les deux copies en mosaïque d'un portrait royal certai-

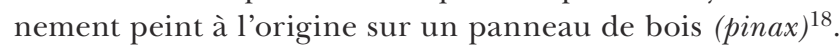
Dans la zone des palais lagides, le pavement aux lutteurs présente des raffinements chromatiques rendus par de fines tesselles de faïence et de verre ${ }^{19}$. Pour effacer encore davantage la discontinuité des éléments et pour aviver la couleur des pavements, on peint la surface des mosaïques après la pose des éléments et, éventuellement, leur ponçage : on reconnaît ainsi des traces de peinture aussi bien sur les joints interstitiels que sur la surface des tesselles, comme le montrent des fragments d'étage de la maison de Fourni à Délos : la peinture rouge, jaune et bleue est visible sur les joints de mortier entre les tesselles de couleur correspondante (fig. 4). La même constatation a été faite pour un pavement hellénistique de Thmouis décoré d'une composition illusionniste de losanges, dont tous les éléments étaient peints ${ }^{20}$. Ainsi, le plus souvent, la couleur est ajoutée après la

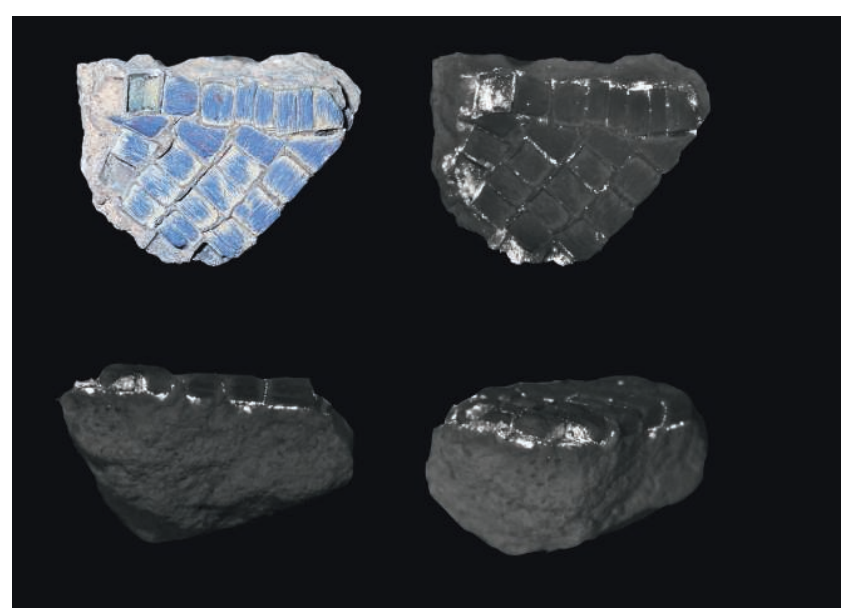

Fig. 5. Délos, fragment d'étage, maison, photographie en lumière normale et en luminescence infrarouge. (C) A. Guimier/ArScAn. pose des tesselles ou des minuscules éléments du vermiculatum. Dans d'autres cas, les pigments sont mélangés à la dernière couche de mortier (bain de pose), qui, lorsqu'on y enfonce les tesselles, remonte dans les joints avec la couleur : des photographies en luminescence infrarouge (VIL en anglais) ont permis de mettre en évidence ce procédé grâce à l'emploi du bleu égyptien, notamment sur un autre fragment d'étage de la maison de Fourni à Délos ${ }^{21}$ (fig. 5).

Selon l'usure du pavement puis les conditions de son enfouissement et de son nettoyage après la découverte et l'exposition dans les musées, la faïence et certains types de verre sont très altérés, et ont perdu une grande partie de leur couleur qu'on ne peut détecter qu'à partir de celles des joints peints. Il n'en reste pas moins vrai qu'il faut en tenir compte pour obtenir une meilleure idée de l'état originel de ces pavements d'époque hellénistique. À partir d'observations à l'œil $\mathrm{nu}$, nous avions entrepris cette recherche, M.-D. Nenna et moi-même, sur des panneaux de Délos (fig. 6 et 7) et d'Alexandrie $^{22}$. Nous la poursuivons maintenant sur le grand panneau figurant la bataille d'Alexandre et de Darius, découvert dans la Maison du Faune à Pompéi et exposé dans le Musée archéologique national de Naples. Les traces de couleur visibles à l'œil nu sur les joints de mortier montrent notamment que les deux principaux protagonistes portaient des vêtements plus colorés qu'on ne le voit aujourd'hui. On peut en proposer un essai de première restitution ${ }^{23}$ (fig. 8 a-b), mais le travail est en cours. 

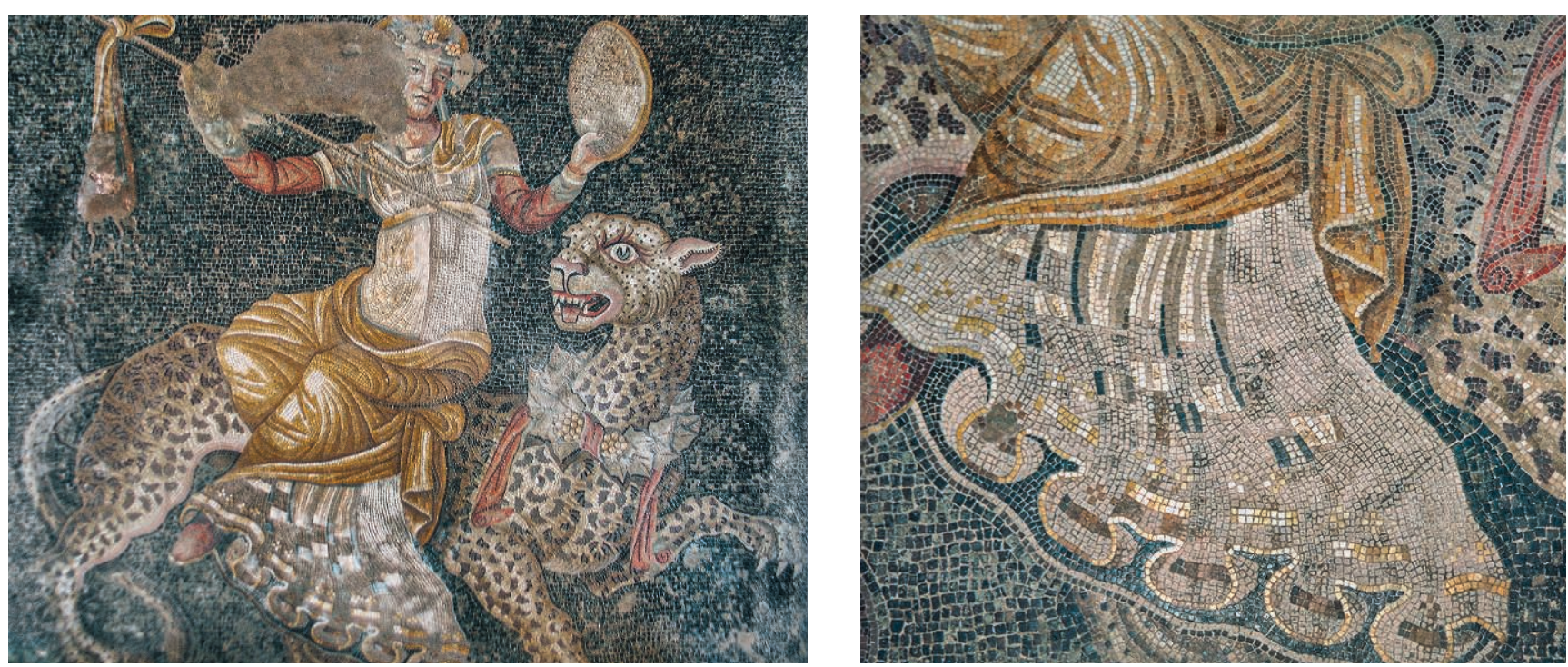

Fig. 6. Délos, maison des Masques, emblema au Dionysos, état actuel. @ A A.-M. Guimier-Sorbets/ArScAn.
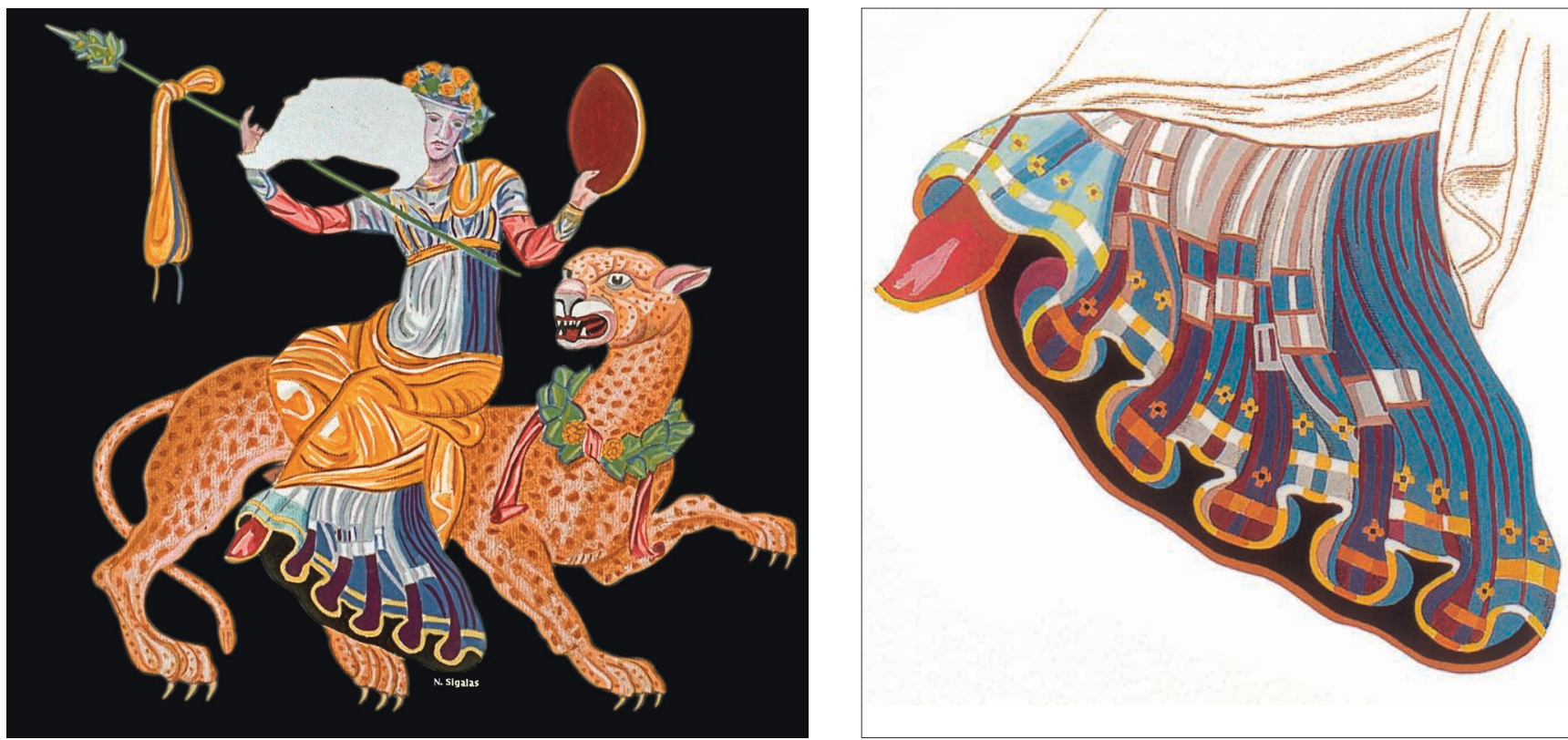

Fig. 7. Délos, maison des Masques, emblema au Dionysos, schéma de restitution des couleurs. Aquarelles N. Sigalas, EFA. 

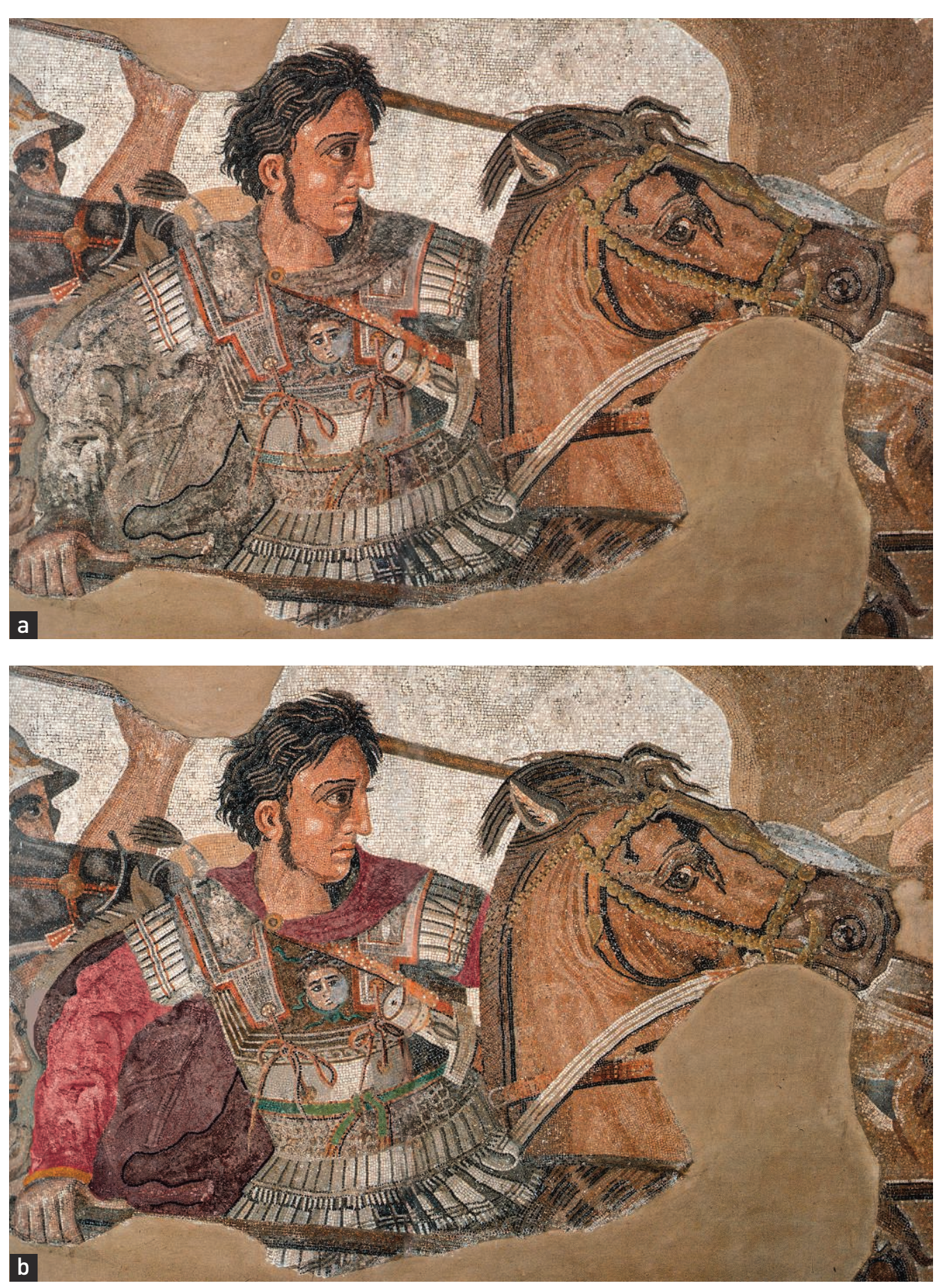

Fig. 8 a-b. Pompéi, détail de la mosaïque d'Alexandre et Darius. a. État actuel ;

b. Proposition de restitution de couleurs. (c) A. Guimier,

A.-M. Guimier-Sorbets/ ArScAn.

\section{Notes}

1. Guimier-Sorbets, 2010, p. 30

2. Robinson, Graham, 1938, p. 171, 174 et tableau en face de la p. 184

3. Guimier-Sorbets, 2001, p. 285.

4. Guimier-Sorbets, 2010, p. 30, avec les références aux diverses publications de fouilles.
5. Rothé, Boislève, Barberan, 2017, p. 51, fig. 9 .

6. Guimier-Sorbets, 2015a, p. 244-246, fig. 5 .

7. Lazaridis, 1993.

8. Coquinot, Pagès-Camagna, Vandenberghe, 2011, p. 524.

9. Guimier-Sorbets, 2016, p. 222-223, fig. 1-2 ; Guimier-Sorbets, 2017 (à paraître).
10. Bruneau, 1972, $\mathrm{n}^{\text {os }} 326,327,339$ sans mention de la peinture ; Vassal, 2006 ; pour la peinture sur ces sols : GuimierSorbets, 2017 (à paraître), fig. 15.

11. Robinson, 1930, p. 55-59, fig. 153161, pl. I ; voir aussi Guimier-Sorbets, 2015b, p. 137, pl. VII.

12. Tiliopoulou Kladouri, Kamatakis, 2009, et p. 130-143 (photos). 
13. La tombe est encore inédite.

Guimier-Sorbets, 2017 (à paraître).

14. Boraik, Guimier-Sorbets, 2013 ;

Guimier-Sorbets, Redon, 2017.

15. Brecoulaki, 2006a et b ; Villard, 2006.

16. Guimier-Sorbets, 2007.

17. Guimier-Sorbets, 2007, p. 206-207, fig. 3 .

18. Pour une explication plus détaillée, voir Guimier-Sorbets, 2019, p. 43-47, fig. 26, 31

19. Guimier-Sorbets, 2005

20. Pour Délos : Guimier-Sorbets, Guimier, 2019 ; pour Thmouis : Rapilliard, Tawfick, Guimier-Sorbets, 2017 (à paraître).

21. Guimier-Sorbets, 2016, p. 224, fig. 4.

22. Guimier-Sorbets, Nenna, $1992 ; 1995$.

23. Guimier-Sorbets, 2016, p. 226, fig. 5-6 et pl. 7 .

\section{Bibliographie}

Boraik M., Guimier-Sorbets A.-M., 2013, "Ptolemaic Baths in front of Karnak temples - Recent Discoveries (season 2009-2010), Part. 2. The floor decoration and painted plaster of the baths", Cahiers de Karnak 14, p. 47-77.

Brecoulaki H., 2006a, La Peinture funéraire de Macédoine. Emplois et fonctions de la couleur, $I V^{e}-I I^{e}$ s. avant J.-C., National Hellenic Research Foundation, Mélétèmata 48, KERA, Athènes.

Brecoulaki H., 2006b, « Considérations sur les peintres tétrachromatistes et les colori austeri et floridi. L'économie des moyens picturaux contre l'emploi de matériaux onéreux dans la peinture ancienne ", dans A. Rouveret, S. Dubel, V. Naas (dir.), Couleurs et matières dans l'Antiquité. Textes, techniques et pratiques (Études de Littérature Ancienne 17), Éditions Rue d'Ulm, Paris, p. 29-42.

Bruneau P., 1972, Les Mosaïques, Exploration archéologique de Délos, XXIX, Athènes, $\mathrm{n}^{\text {os }} 326,327,339$.

Coquinot Y., Pagès Camagna S., Vandenberghe Y., 2011, «Fragment de sol de couleur rouge, contribution à l'étude matérielle du fragment ", dans Cat. Exp. Paris, 2011, Au Royaume d'Alexandre le Grand, la Macédoine antique [Exp. Paris, musée du Louvre, 13 octobre 2011-16 janvier 2012] S. Descamps-Lequime,

K. Charatzopoulou (dir.), musée du Louvre Éditions/Somogy Éditions, Paris, p. 524 et fig. 21, p. 85.

Guimier-Sorbets A.-M., 2001, « Les ateliers de mosaïques à Alexandrie à l'époque hellénistique et au début de l'époque impériale : continuité et innovation ", dans D. Paunier, C. Schmidt (dir.), La mosaïque gréco-romaine VIII : Actes du VIII Colloque international pour l'étude de la mosaïque antique et médiévale [Lausanne, Suisse, 6-11 octobre 1997], Cahiers d'archéologie romande, Lausanne, p. 282-297.

Guimier-Sorbets A.-M., 2005, « La mosaïque aux lutteurs de la Bibliotheca
Alexandrina. Techniques picturales et mosaïstiques dans les palais

d'Alexandrie ", Musiva E' Sectilia, International Journal for the Study of Ancient Pavements and wall revetments in their decorative and architectural context, Pise, I, p. 15-34.

Guimier-Sorbets A.-M., 2007, « De la peinture à la mosaïque : problèmes de couleurs et de techniques à l'époque hellénistique ", dans S. DescampsLequime (dir.), Peinture et couleur dans le monde grec antique, musée du Louvre Editions/Somogy Éditions, Paris, p. 204-217.

Guimier-Sorbets A.-M., 2010, «Peindre les sols : quelques emplois attestés dans le monde grec ", dans I. Bragantini (dir.), Atti del X Congresso internazionale AIPMA (AION Quad. 18), Naples, p. 29-40 pl. III-IV.

Guimier-Sorbets A.-M., 2015a, « Les tombes monumentales du royaume odryse : des modèles macédoniens ?", dans Cat. Exp. Paris, 2015, L'épopée des rois thraces des guerres médiques aux invasions celtes, 479-278 avant J.-C. : découvertes archéologiques en Bulgarie [Exp. Paris, musée du Louvre, 16 avril-20 juillet 2015], J.-L. Martinez et al. (dir.), musée du Louvre Editions/Somogy Editions, Paris, p. 244-246.

Guimier-Sorbets A.-M., 2015b, « Le décor architectural grec en Thébaïde : pavements et peintures murales dans des bains de l'époque lagide ", dans A. Marangou, G. Gorre (dir.), La présence grecque dans la Vallée de Thèbes, Presses universitaires de Rennes, Rennes, p. 135-138, pl. IV-VIII.

Guimier-Sorbets A.-M., 2016, « Ajouter de la couleur aux mosaïques à l'époque hellénistique : emplois de peinture et de mortiers colorés ", dans L. Neira Jimenez (dir.), Estudios sobre mosaicos antiguos y medievales, Actas del XIII Congreso international de la AIEMA (Madrid, 14-18 septembre 2015), Hispania Antigua, Serie Arqueologica 6, Journal of Roman archaeology, Rome, p. 222-228, pl. 7.

Guimier-Sorbets A.-M., 2017 (à paraître), «Les sols de mortier en Grèce et en Égypte à l'époque hellénistique : technique et décor ", Actes du colloque Pavements et sols en mortier : vocabulaire, techniques, diffusion (Aix-en-Provence, avril 2017).

Guimier-Sorbets A.-M., 2019, Mosaïques d'Alexandrie, pavements d'Égypte grecque et romaine (Antiquités Alexandrines 3), Centre d'Études Alexandrines, Alexandrie.

Guimier-Sorbets A.-M., Guimier A., 2019, «Quand et comment tenter de reproduire les couleurs antiques du décor architectural : études de cas de mosaïques et de peintures », Actes du colloque international Reconstruction of Polychromy, Restituer les couleurs (Bordeaux, 29 novembre-1 ${ }^{\text {er }}$ décembre 2017), Ausonius, Archéovision 8, p. 15-23.

Guimier-Sorbets A.-M., Nenna M.-D., 1992, «L'emploi du verre, de la faïence et de la peinture dans les mosaïques de Délos », BCH 116, p. 607-631.

Guimier-Sorbets A.-M., Nenna M.-D., 1995, «Réflexions sur la couleur dans les mosaïques hellénistiques : Délos et Alexandrie ", BCH 119, p. 529-563.

Guimier-Sorbets A.-M., Redon B., 2017, "The floors of the Ptolemaic baths of Egypt: between technique and aesthetic", dans B. Redon (dir.), Collective baths in Egypt 2. New discoveries and perspectives, Études Urbaines 10, IFAO, Le Caire, p. 139-170.

Lazaridis D., 1993, Amphipolis, TAPA, Athènes, p. 145, fig. 108 (édition en grec).

Rapilliard M., Tawfick H., Guimier-Sorbets A.-M., 2017 (à paraître), "A mosaic from Thmuis Egypt: continuation of a conservation project and complementary observations regarding the constituent materials", Actes du $13^{e}$ Colloque international de l'ICCM (Barcelone, octobre 2017).

Robinson D. M., 1930, Excavations at Olynthus, Part II. Architecture and Sculpture, Baltimore.

Robinson D. M., Graham J. W., 1938, Excavations at Olynthus, Part VIII. The Hellenic house. A study of the houses found at Olynthus with a detailed account of those excavated in 1931 and 1934, Baltimore.

Rothé M.-P., Boislève J., Barberan S., 2017, «La maison de la Harpiste et son décor à Arles (Bouches-du-Rhône). Nouvelles données sur l'occupation tardorépublicaine d'Arelate », Gallia 74, p. 43-76.

Tiliopoulou M., Kladouri N., Kamatakis P., 2009, "Study for conservation of the floors of the Palace of Aegae", dans A. Kottaridi (dir.), The Palace of Aegae 20072009, The commencement of a major project, Ministry of Culture and Tourism. 17th Ephorate of Prehistoric and Classical Antiquities, Thessalonique, p. 64-73.

Vassal V., 2006, Les pavements d'opus signinum. Technique, décor, fonction architecturale, BAR-IS 1472, Oxford.

Villard L., 2006, « L'essor du chromatisme $\mathrm{au} \mathrm{IV}^{\mathrm{e}}$ siècle. Quelques témoignages contemporains ", dans A. Rouveret, S. Dubel, V. Naas (dir.), Couleurs et matières dans l'Antiquité. Textes, techniques et pratiques (Études de Littérature Ancienne 17), Éditions Rue d'Ulm, Paris, p. 43-53. 